\title{
La competencia mediática de las personas mayores andaluzas: retos para una inclusión social plena
}

\author{
Paloma Contreras-Pulido \\ Universidad de Huelva \\ paloma.contreras@uhu.es \\ Rafael MARFIL-CARMONA \\ Universidad de Granada \\ rmarfil@ugr.es \\ Juana Ma ORTEGA \\ Universidad de Jaén \\ jmortega@ujaen.es
}

\section{Resumen:}

Las personas mayores dentro de la sociedad actual representan un porcentaje elevado y que tiende a ser mayor con el paso de los años. Además, estas personas mayores son cada vez más activas dentro de dicha sociedad, participando incluso de los avances derivados de la utilización de las tecnologías digitales. En el presente trabajo se trata de analizar el nivel de alfabetización mediática de las personas mayores de 55 años en la Comunidad Autónoma Andaluza.

Palabras Claves: Competencia mediática; mayores; Andalucía; alfabetización mediática; inclusión social.

\section{The media competence Andalusian seniors: challenges for full social inclusion}

\begin{abstract}
:
Older people in current society represent a high percent age that tends to be higher over the years. In addition, these seniors are becoming more active within this society taking part of even the developments resulting from the use of digital technologies. This paper analyzes the level of media literacy of people over 55 years in the Autonomous Community of Andalusia.
\end{abstract}

Key Words: Media competence; seniors; Andalusia; media literacy; social inclusion.

\section{Referencia normalizada:}

Contreras Pulido, P., Marfil Carmona, R. y Ortega, J.M. (2014): La competencia mediática de las personas mayores andaluzas: retos para una inclusión social plena. Historia y Comunicación Social. Vol. 19. Núm. Especial Enero. Págs. 129-142.

Sumario: 1. Introducción. 2.¿Qué significa ser mayor hoy? 3. La competencia mediática en personas mayores 4. Objetivos y metodología. 4.1. Población y muestra. 4.2. El Cuestionario. 5. Resultados y discusión. 5.1. Redes. 5.2. Consumo mediático y opinión propia. 5.3. Aspectos formales y estéticos. 6 . Conclusiones. 


\section{Introducción}

Ser ciudadano activo hoy día requiere, entre otras muchas cuestiones, de la utilización de las tecnologías digitales. Los mayores tienen hoy una oportunidad de ser partícipes de las transformaciones sociales también a través de estos medios, alejándose así de cualquier forma de exclusión individual o social. Hay que tener en consideración que, durante las próximas décadas aumentará notablemente el sector de la población mayor de 55 años en el mundo. En este artículo se exponen los resultados del proyecto de investigación ALFAMED ${ }^{1}$ tras finalizar la recogida y análisis de datos a través del cuestionario que, en 2012 y 2013, ha evaluado el nivel de alfabetización mediática de las personas mayores de 55 años en la Comunidad Autónoma de Andalucía (España), incluyendo a las que participan en aulas universitarias de mayores y a las que no tienen vinculación universitaria.

\section{2. ¿Qué significa ser mayor hoy?}

El término "mayor" y, por extensión, el concepto de envejecimiento, debería vincularse a la implicación social y a la capacidad para desarrollar una ciudadanía activa. El término vejez ha pasado de su acepción biológica a una perspectiva más social. García Mínguez (2004: 43), asegura que "del jubilado improductivo hemos llegado al concepto de mayor competente e imaginativo", explicando así la importancia de la educación a lo largo de la vida.

Según los datos ofrecidos en 2012 por el Instituto Nacional de Estadística (INE) ${ }^{2}$, el 37\% de la población española será mayor de 64 años en 2052 debido al envejecimiento de la pirámide poblacional. En Europa, uno de cada cuatro europeos tendrá más de 65 años en 2050, según el último informe de la Organización Mundial de la Salud (OMS) (2012).

Actualmente, que una persona mayor goce de un buen estado de bienestar supondrá que, además de estar sano físicamente, también ha de estarlo emocional y psicosocialmente. Se trata, en resumen, de enfocar esta etapa a la calidad de vida (Pérez Serrano, 2004: 36). Esos factores tendrán que ver con la forma en la que nos relacionamos con los demás. Los medios de comunicación y las tecnologías de la sociedad de la información son factores claves de esa relación. Las competencias mediáticas pueden convertirse en herramientas para mejorar la vida social.

\footnotetext{
Proyecto de Excelencia de la Junta de Andalucía (P10SEJ-5823) «La competencia audiovisual de la ciudadanía andaluza. Estrategias de alfabetización mediática en la sociedad del ocio digital».

2 Informe «Proyección de la Población de España a Largo Plazo, 2012-2052» publicado por el INE en diciembre de 2012. Disponible enhttp://www.ine.es/jaxi/menu.do?type=pcaxis\&path=\% $2 \mathrm{Ft} 20 \%$ 2Fp251\&file=inebaseConsultado el 1 de abril de 2013.
} 


\section{La competencia mediática en personas mayores}

Evaluar el nivel de competencia mediática con el que cuenta la ciudadanía es, actualmente, una prioridad para estados e instituciones supranacionales como el Parlamento y la Comisión Europa, entre otras, interesadas en conocer hasta qué punto la población es competente y así elaborar medidas a favor del desarrollo de la alfabetización mediática. Este concepto incluye la interacción con los medios de comunicación, tanto en soportes audiovisuales, escritos como digitales.

En ese contexto, el profesor de la Universidad Pompeu Fabra, Joan Ferrés, y un amplio equipo de expertos desarrollaron en 2007 el documento «La competencia en comunicación audiovisual: propuesta articulada de dimensiones e indicadores» (2007), proponiendo el siguiente concepto de competencia mediática:

la capacidad de un individuo para interpretar y analizar desde la reflexión crítica las imágenes y los mensajes audiovisuales, y para expresarse con una mínima corrección en el ámbito comunicativo. Esta competencia está relacionada con el conocimiento de los medios de comunicación y con el uso básico de las tecnologías multimedia necesarias para producirla. (Ferrés, 2007: 102).

Ferrés (2007) elaboró una serie de dimensiones e indicadores útiles para evaluar la competencia mediática, que no deben entenderse de manera individual, sino desde la relación que hay entre ellas. La investigación de Alfamed ha contemplado esa visión de conjunto. Los cuestionarios, tal y como se explica en la metodología, contienen cada una de las seis dimensiones de la competencia mediática: lenguaje, tecnología, producción y difusión, recepción e interacción, ideología y valores y estética (Ferrés, 2007: 103).

Centrándonos el caso de las personas mayores, según Santibáñez y varios (2012), las mayores de 65 años son vulnerables ante los medios por varios motivos. En primer lugar, no son personas nativas digitales, sino que son inmigrantes digitales (Prensky, 2001), desconociendo el funcionamiento de los medios audiovisuales. Además, se convierten en consumidores pasivos cuando están delante de la televisión, de cualquier pantalla, escuchando la radio o visualizando la publicidad exterior.

Según el último informe "La sociedad de la información", publicado por la Fundación Telefónica, en España "el grupo de edad que más crece en lo relativo al acceso a Internet es el que aglutina a personas entre 55 y 64 años, que pasan del 37,7\% al 43,7\%"(Sociedad de la información, 2013: 33). Otros datos del estudio, sin embargo, refrendan la existencia de una importante brecha digital: "Mientras que un $96 \%$ de jóvenes entre 16 y 24 años han utilizado Internet en los últimos tres meses tan sólo el 18\% de los mayores de 65 años ha accedido en el mismo periodo" (Fundación Telefónica, 2013: 36).

Es la adaptación a una época compleja, que constituye una revolución tan relevante como fue la industrialización o la imprenta (Scolari, 2008). Realizar un diagnóstico para mejorar la competencia mediática y digital ha sido el eje central de la investigación cuyos objetivos se exponen en este texto. 


\section{Objetivos y metodología}

Los objetivos del proyecto Alfamed se centrann en detectar las necesidades y carencias de la población andaluza mayor de 55 años en relación con los niveles de competencia en comunicación: tecnología, lectura crítica y producción audiovisual, diagnosticando su grado de competencia mediática.

Para ello se utilizan metodologías descriptivas, que son, tal y como asegura Hernández Pina (1997), las más adecuadas para el desarrollo de una evaluación. Para conocer un fenómeno social, es básico analizar, observar y preguntar (Corbetta, 2003). Así, la investigación se basa en técnicas cuantitativas de recogida de datos, con el cuestionario como herramienta principal, complementadas con otros procedimientos cualitativos, como la entrevista en profundidad o los grupos de discusión (focus group). Este artículo se centra en los resultados obtenidos a través de los cuestionarios administrados a una muestra representativa de personas mayores de 55 años en todas las provincias de Andalucía.

\subsection{Población y muestra}

La población del estudio la conforman las personas mayores de 55 años de la comunidad autónoma andaluza. Se seleccionó una muestra de 479 personas de las 8 provincias andaluzas, con una media de 59,9 personas por provincia. De las 479 personas, 245 pertenecían a alguno de los Programas Universitarios de Mayores implementados en las diferentes universidades andaluzas.

La franja de edad más numerosa la componen las personas entre 55 y 60 años, constituyendo un $31,7 \%$ de la muestra seleccionada (152 sujetos). La franja de edad con menos representantes es la de mayores de 80 años, con 10 sujetos $(2,1 \%$ de la muestra total).

La muestra respeta la proporcionalidad poblacional por sexo. En Andalucía, hay más mujeres que hombres mayores de 55 años. La población andaluza femenina mayor es de $54,97 \%$ en 2013 , lo que se refleja en un $60,3 \%$ en la muestra de la investigación.

\subsection{El cuestionario}

Consta de 28 preguntas. Tras las cuestiones de carácter socio-demográfico (edad, etc.) que van desde la pregunta 1 a la 11 , las dimensiones correspondientes a la competencia mediática se han estructurado según la Tabla 1. 
Tabla 1. Estructura del cuestionario atendiendo a las dimensiones de la Competencia Mediática.

\begin{tabular}{|c|c|}
\hline Dimensión & Preguntas \\
\hline \multirow[t]{3}{*}{ Lenguaje } & $\begin{array}{l}\text { 11. Valore de } 1 \text { a } 5 \text { la importancia que tienen en su quehacer } \\
\text { cotidiano los siguientes medios }\end{array}$ \\
\hline & 12. Señale si ha realizado alguna de estas actividades \\
\hline & 13. En caso de que utilice internet, ¿para qué lo hace? \\
\hline \multirow[t]{2}{*}{ Tecnología } & $\begin{array}{l}\text { 14. Indique con la frecuencia con la que realiza las siguientes } \\
\text { actividades }\end{array}$ \\
\hline & 15. ¿Con qué aplicaciones informáticas ha trabajado? \\
\hline \multirow[t]{4}{*}{$\begin{array}{l}\text { Producción y } \\
\text { programación }\end{array}$} & $\begin{array}{l}\text { 16. Para crear su propia opinión de las noticias, } \\
\text { acontecimientos... ¿qué hace? }\end{array}$ \\
\hline & $\begin{array}{l}\text { 17. ¿Alguna vez se ha puesto en contacto con un programa de } \\
\text { TV, radio, prensa, revista o página web? }\end{array}$ \\
\hline & $\begin{array}{l}\text { 18. ¿Utiliza Internet para cooperar con un grupo de ciudadanos } \\
\text { en actividades sociales o culturales? }\end{array}$ \\
\hline & $\begin{array}{l}\text { 19. ¿Antes de introducir sus datos personales (tarjeta de } \\
\text { crédito, correo electrónico, teléfonos, etc.) en internet, a la } \\
\text { hora de hacer una compra, descargarse algún programa, o } \\
\text { registrarse en un servicio electrónico? }\end{array}$ \\
\hline \multirow[t]{3}{*}{$\begin{array}{l}\text { Recepción e } \\
\text { interacción }\end{array}$} & $\begin{array}{l}\text { 20. ¿Ha utilizado internet para comunicarse con las } \\
\text { autoridades públicas? }\end{array}$ \\
\hline & $\begin{array}{l}\text { 21. ¿Ha recurrido en este último año a las instancias oportunas } \\
\text { para denunciar el incumplimiento de normas en materia } \\
\text { audiovisual? }\end{array}$ \\
\hline & $\begin{array}{l}\text { 22. ¿Se ha cuestionado alguna vez si se le ocultaba parte de } \\
\text { una información? }\end{array}$ \\
\hline \multirow[t]{2}{*}{ Ideología y valores } & $\begin{array}{l}\text { 23. ¿Ha colaborado usted alguna vez en la creación de una } \\
\text { campaña (o en su difusión), mediante el envío de correos } \\
\text { a toda su lista de conocidos que cuestionen los valores o } \\
\text { estereotipos presentes en alguna producción mediática } \\
\text { (anuncio, campaña publicitaria, serie de TV, etc.)? }\end{array}$ \\
\hline & $\begin{array}{l}\text { 24. ¿Utiliza usted algún medio (e-mail, blog, etc.) para mandar } \\
\text { mensajes, o realizar acciones, que contribuyan a la mejora del } \\
\text { entorno social en el que vive? }\end{array}$ \\
\hline \multirow{4}{*}{ Estética } & $\begin{array}{l}\text { 25. A la hora de decidirse por un medio, ¿tiene en cuenta solo } \\
\text { lo que comunica o en esa elección le da valor a los aspectos } \\
\text { formales y estéticos? }\end{array}$ \\
\hline & $\begin{array}{l}\text { 26. A nivel de aspectos formales o estéticos ¿ha rechazado } \\
\text { usted algún medio por no tener un mínimo de calidad estética? }\end{array}$ \\
\hline & $\begin{array}{l}\text { 27. ¿Considera que un mismo mensaje puede ser más o menos } \\
\text { efectivo/eficaz si se refuerzan los aspectos estéticos? }\end{array}$ \\
\hline & $\begin{array}{l}\text { 28. ¿Qué medio de comunicación utilizaría por sus } \\
\text { posibilidades estéticas? }\end{array}$ \\
\hline
\end{tabular}

Fuente: Elaboración propia 


\section{Resultados y discusión}

Las respuestas obtenidas son interesantes con respecto a la relación de los mayores con las tecnologías, el uso de Internet y de sus opciones para la participación y relación, comprendiendo las tecnologías actuales como TRIC (Tecnologías+Relación+Información+Comunicación) en lugar de TIC (Gabelas, Marta-Lazo y Aranda, 2012). Además, los resultados de esta investigación son un diagnóstico sobre los hábitos de consumo mediático de las personas mayores,tanto en el acceso a la información como en la formación de una opinión propia.

En el apartado de consumo tecnológico, centrado en equipos informáticos utilizados, 189 personas aseguran usar el correo electrónico, siendo el tipo programa más utilizado, con un 39,46\%, seguido por Word y OpenOffice, con el que están familiarizadas 69 personas, lo que supone un 14,41\% del total. El resto de programas, como Photoshop, Picasa y hojas de cálculo, entre otros, registran valores testimoniales, mientras que un $18,58 \%$ asegura no conocer ninguna aplicación informática.

\subsection{Redes}

Casi la mitad de las personas encuestadas, el $45,72 \%$, afirma que utiliza Internet para buscar información. El 22,34\% asegura que lo utiliza con un fin social, para comunicarse con otras personas. Es también significativo que un $21,50 \%$ afirma no usar la web. Además, el segmento poblacional encuestado muestra reticencias a realizar transacciones comerciales a través de la Red: solo el 1,04\% afirma utilizarlo para la compra o reserva de productos o servicios. El 70,15\% nunca ha comprado por Internet y el $45,51 \%$ de las personas encuestadas no confiaría en ningún sitio en el que tuviera que introducir sus datos personales. En el ámbito audiovisual, destaca que el 77,45\% nunca ha subido archivos a YouTube.

En lo relativo a las redes sociales, la herramienta menos utilizada es Tuenti, en la que un $83,72 \%$ de los encuestados afirma no haber entrado nunca, algo lógico por el perfil de esta red social. Sin embargo, uno de los canales que, en principio, parecen más apropiados para la aportación de la experiencia por parte de las personas mayores, como es el blog, está absolutamente infrautilizado, según los resultados de la investigación: un $80,38 \%$ afirma no haberlo utilizado nunca. Otra herramienta de carácter conceptual, más parecida a los formatos tradicionales de acceso al conocimiento, sería la Wikipedia, en la que el índice de uso frecuente es muy bajo, con un $6,47 \%$ frente al $24,63 \%$ que la han utilizado alguna vez.

La red más utilizada es Facebook, con un 16,08\% que asegura usarla con frecuencia. No obstante, más de la mitad de las personas encuestadas, el $55,11 \%$, nunca ha entrado en ella. Otras posibilidades, como el uso del chat, con un $4,38 \%$ de uso frecuente, tienen un valor testimonial en las rutinas cotidianas de la muestra encuestada, aunque no desconocen sus posibilidades o funcionamiento, ya que el $21,92 \%$ asegura haber chateado alguna vez. El cuestionario confirma el bajo grado de aprovechamiento por parte de la población mayor de 55 años, tal y como indica el gráfico 1. 
Gráfico 1. Uso de Internet por parte de las personas mayores de 55 años.

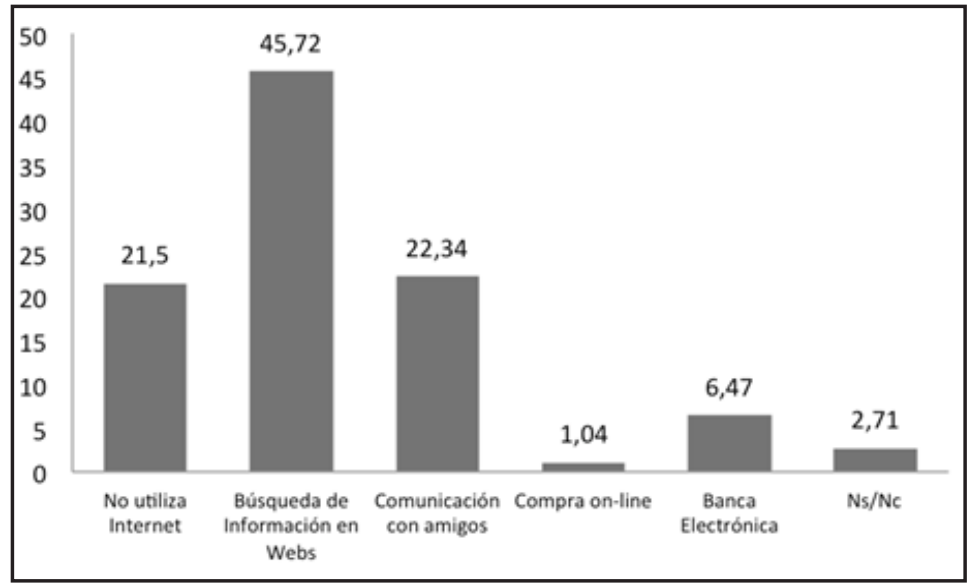

Fuente: Elaboración propia.

Es relevante comparar el acceso a Internet con otro tipo de actividades que realizan las personas mayores encuestadas, valorando así si se trata de un porcentaje bajo de participación en la Red o un grado mejorable de implicación en otro tipo de actividades en su entorno e implicación ciudadana. En este sentido, mientras que el 44,05\% usa Internet a diario, no es superior el porcentaje de personas que lee libros cada día $(33,4 \%)$. Semanalmente, un $63,67 \%$ accede a Internet en algún momento, mientras que un $46,56 \%$ acude al cine o al teatro. El porcentaje de personas interesadas en las propuestas culturales de su entorno es ligeramente inferior al porcentaje de mayores que acceden a Internet, lo que indica que menos de la mitad aprovechan regularmente las posibilidades que tienen a su alcance el envejecimiento activo.

La pregunta sobre el uso de Internet para cooperar con grupos de ciudadanos en actividades sociales o culturales confirma la reducida tendencia en el ejercicio de la ciudadanía activa en la Red, ya que solo el 31,73\% participa en actividades de estas características, de los cuales únicamente el 10,44\% lo hace con frecuencia. El $68,06 \%$ asegura no utilizar Internet con estos fines. Ni siquiera se percibe como una vía para asuntos de consulta o gestión: el 79,54\% de los encuestados afirma no haber utilizado nunca Internet para comunicarse con autoridades públicas, frente al 8,56\% que no lo ha hecho en los últimos tres meses. Los resultados del cuestionario reflejan que más de la mitad, el 51,15\%, nunca ha utilizado ningún medio (mail, blog, etc.) para mandar mensajes o realizar acciones que contribuyan a la mejora de su entorno social.

\subsection{Consumo mediático y opinión propia}

En lo referente a consumo mediático, las personas mayores andaluzas otorgan una mayor importancia a la radio y a la televisión en su quehacer cotidiano, puntuándolas con 3,4 y 3,3 sobre 5 respectivamente, frente al uso menos frecuente de las redes 
sociales, que se sitúa en 1,74 . Por su parte, otros usos de internet $(2,87)$ y la prensa en papel $(2,91)$ obtienen en esta investigación grados intermedios de uso, de igual forma que se consulta diariamente la prensa digital, valorada como 2,43 sobre 5 (Gráfico 2).

Gráfico 2. Importancia de los medios en el quehacer cotidiano de los mayores.

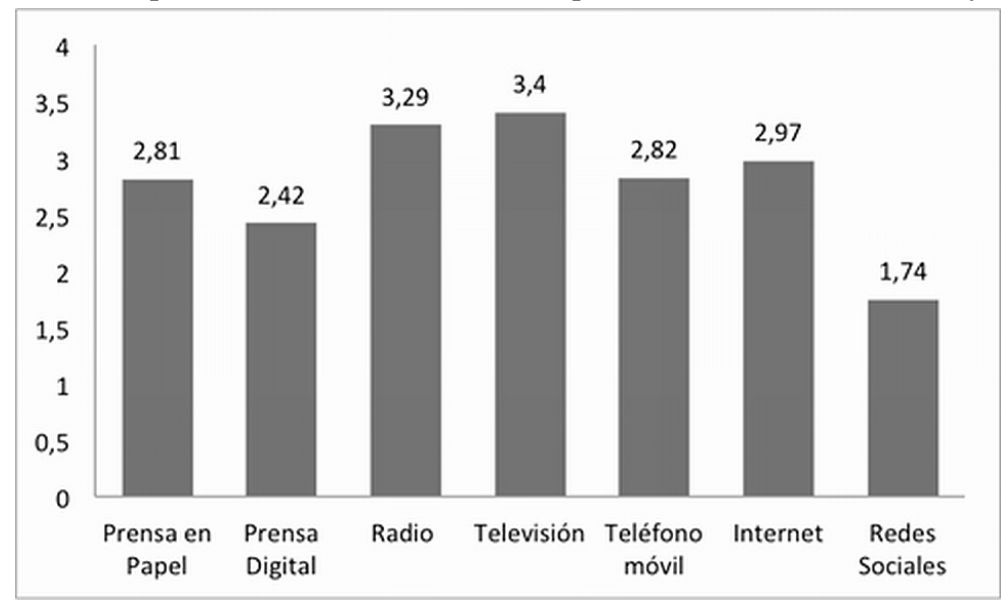

Fuente: Elaboración propia

Para formar su propia opinión, un 53,03\% recurre a los medios tradicionales (televisión, prensa y radio), mientras que un $37,79 \%$ afirma buscar información en otros medios que considera fiables. Casi un $10 \%$ ( $8,98 \%$ de los encuestados) afirma no hacer nada para crear esa propia opinión de las noticias, lo que representa un claro indicador de la actitud frente a la realidad social.

Además, existe un alto grado de desconfianza ante los medios de comunicación, ya que un $67,64 \%$ de los encuestados, es decir, 324 personas, afirman que se han cuestionado alguna vez que se les estaba ocultando parte de una información, frente al $32,15 \%$ que asegura no habérselo planteado nunca. La sospecha ante la veracidad y la transparencia es un claro indicador de la madurez conceptual en lo relativo a los procesos de información.

Sin embargo, un $20,88 \%$, asegura haberse puesto alguna vez en contacto con un programa de Televisión, radio, prensa, revista o página web, lo que indica la implicación activa de los que sí mantienen hábitos de información y expresión.

El grado de interés por el cumplimiento normativo y la ética de los medios es otro de los resultados destacables, ya que un 3,34\% ha recurrido en el último año a las instancias oportunas para denunciar el incumplimiento de normas en materia audiovisual. El dato porcentual no es tan relevante como el total de personas a las que corresponde esta acción, ya que 16 mayores que han ejercido ese derecho.

Internet, sin embargo, es considerada por la mayoría una herramienta para la crítica de los contenidos mediáticos. El 73,07\% afirma que nunca ha colaborado en 
la creación o difusión de una campaña mediante el envío de correos masivos, que cuestionen los valores o estereotipos presentes en alguna producción mediática.

\subsection{Aspectos formales y estéticos}

Algo menos de la mitad de las personas encuestadas, el 48,85\%, dan importancia a los aspectos formales y estéticos. El 58,04\% reconoce que ha rechazado algún medio por no tener un mínimo de calidad estética, reconociendo un alto porcentaje, el 78,91\% (378 personas), la relación directa entre los aspectos formales y la eficacia del mensaje. Incluso a la hora de confiar para realizar transacciones económicas, la desconfianza se atenúa por el aspecto profesional del sitio web para un $16,49 \%$ de las personas encuestadas.

En el terreno de la expresión y el uso de los medios audiovisuales, esas posibilidades estéticas están muy repartidas en la valoración de las personas encuestadas, que utilizarían, en un $22,96 \%$ el vídeo y en un $22,96 \%$ la web atendiendo a esas potencialidades. El periódico sólo es considerado un recurso con potencialidad estética por un $14,41 \%$. Un aspecto destacable es la valoración de la radio como un medio con recursos estéticos por aprovechar (Gráfico 3).

Gráfico 3. Qué medio de comunicación utilizaría atendiendo a sus posibilidades estéticas.

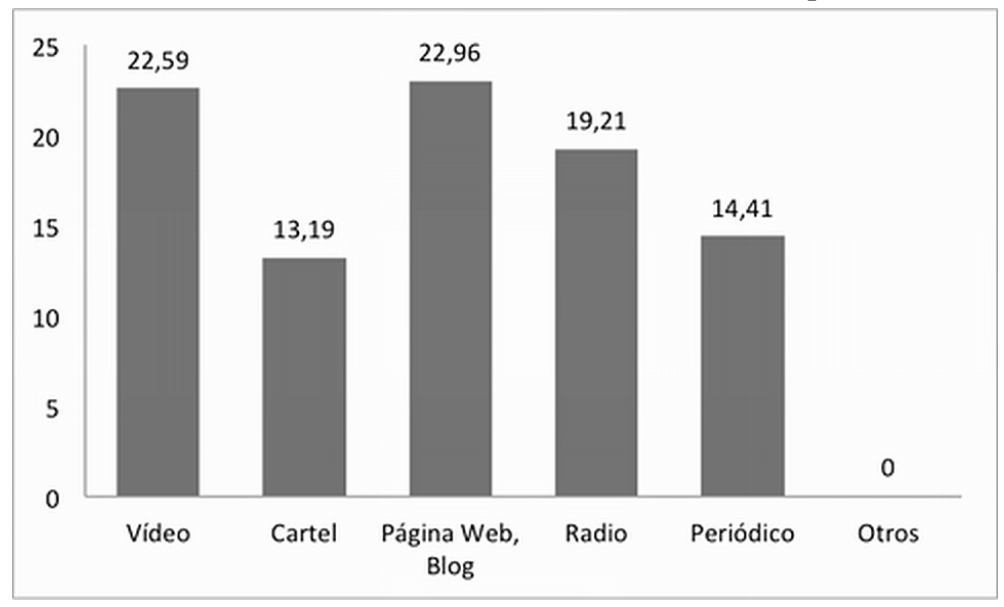

Fuente: Elaboración propia

La síntesis de estos resultados es un uso muy limitado de las tecnologías, en comparación con los recursos disponibles; un acceso moderado a Internet pero poco interactivo; la tendencia dominante de dos de los medios tradicionales (televisión y radio) en los hábitos de consumo mediático y, por último, la valoración de las posibilidades formales y estéticas de los medios. 


\section{Conclusiones}

Los resultados de la investigación de Alfamed constatan que el mero consumo mediático no asegura la capacidad crítica. Una de las conclusiones más importantes de esta investigación es que nos encontramos con una franja poblacional que no es tan activa en el ámbito mediático y digital como en el actual proceso de independencia y modernización que supuestamente está viviendo. Parece estar en proceso de superación el concepto negativo de la vejez propio del pasado. Esa dinámica, sin embargo, debe venir acompañada por la mejora de la competencia mediática, cuyas dimensiones se valoran a continuación atendiendo a los resultados del cuestionario:

En lo que se refiere al dominio de lenguajes, los entornos mediáticos en general y digitales en particular no son vistos con mucha confianza por parte de la población mayor andaluza, aunque se trata fundamentalmente de una visión crítica basada en el desconocimiento de los códigos y las claves narrativas. No se aprecia lo que no se comprende, y eso sucede en un alto porcentaje con los procesos del lenguaje audiovisual y digital.

Vinculada al lenguaje mediático aparece la faceta estética del mensaje. Aproximadamente la mitad de los encuestados reconoce haber realizado una valoración de la estética y de los aspectos formales en los medios, y casi un $80 \%$ son conscientes de la importancia del factor estético. Se tienen en cuenta, por tanto, las potencialidades estéticas de los medios a la hora de hacer uso de ellos, destacando la radio en una generación que no ha recibido tanta influencia de la imagen y la cultura visual como otras franjas poblacionales de menor edad. Conocer posibilidades expresivas de los mensajes audiovisuales puede ser una vía para la participación creativa y crítica a través de la Red.

Sobre la dimensión tecnológica, hay un nivel básico de conocimiento de las herramientas informáticas: word, correo, etc., resultado de una trayectoria de más años en la concienciación formativa antes de la revolución 2.0. Sin embargo, en ningún caso supera el $50 \%$ y cerca de un $20 \%$ no conoce ninguna aplicación informática. Esa situación constituye una oportunidad para impulsar y divulgar las TIC a través de acciones formativas, así como un reto para las diferentes instituciones que atienden a este colectivo.

La dimensión que hace referencia a la recepción e interacción es la que nos lleva directamente a valorar la denominada dieta mediática, es decir, los hábitos de lectura y consumo de medios, pero también la interacción y participación. En la primera vertiente, la analítica, más vinculada a la selección y evaluación de medios, cerca de la mitad de las personas mayores encuestadas en esta muestra recurre a medios tradicionales para informarse (televisión, radio). Los hábitos informativos sí marcan una diferencia generacional.

Además, destaca que un 10\% de las personas encuestadas muestra una actitud de apatía frente a las posibilidades informativas, ya sea por desinterés o por desconfianza. Esto puede interpretarse como una actitud extrema que, seguramente, encuen- 
tra una correlación en otros ámbitos de la vida, siendo afortunadamente un porcentaje minoritario las personas que muestran esta falta de interés.

Es destacable el alto grado de desconfianza mostrado ante los medios $(67,64 \%)$ en lo que se refiere a la veracidad que les otorgan. Esa visión crítica, quizá excesiva, es rotundamente desmitificadora, y puede ser resultado de la experiencia vital más que al desarrollo de competencias analíticas. El reto en este sentido, sería convertirse en personas conscientes de la manipulación pero que, por ello, contrastan noticias y no renuncian a estar informadas. Desde el punto de vista educativo, esta desconfianza puede contemplarse como un punto de partida para desarrollar la capacidad crítica y expresiva, a través de la profundización en estrategias de alfabetización audiovisual.

Dentro de esas dimensiones de la competencia digital, la interacción supone un estadio más avanzado con respecto al consumo mediático. Se trata de conseguir una implicación activa en el entorno a través de los procesos de comunicación y los medios que las personas mayores tienen hoy a su alcance. En esa vertiente relacional existe un grado bajo de aprovechamiento de las redes sociales y de las posibilidades de Internet, que es utilizada por la mitad de las personas encuestadas con un fin básicamente informativo. Incluso la faceta informativa también adolece de los criterios fundamentales, ya que se desconocen las posibilidades de laWikipedia o de herramientas como el blog. Destaca, además, la desconfianza ante la actividad comercial y económica en la web.

Hay una amplia gama de posibilidades formativas por implementar en el ámbito audiovisual y digital. Casi el $80 \%$ de las personas encuestadas nunca han subido contenidos de vídeo a YouTube, por lo que es necesario combinar la enseñanza del lenguaje audiovisual y de su difusión en soportes digitales.

Facebook es la red más utilizada. Aun así, más de la mitad de las personas mayores encuestadas nunca ha hecho uso de esta red, por lo que se debe impulsar la comprensión de las posibilidades de las redes.

Un porcentaje reducido ha utilizado los canales existentes a su disposición para reclamar algún derecho como usuario de los medios de comunicación. Es relevante que 16 mayores reclamaran el incumplimiento de normas en materia audiovisual durante el último año. A pesar de este dato, es posible que se trate de ejercicios de ciudadanía activa fuera de la red, ya que Internet no se contempla como una vía para solucionar problemas, según los resultados de la investigación.

La faceta expresiva, vinculada a la producción de contenidos, encuentra un factor positivo en la consideración de los valores estéticos y formales de los medios, entre los que se incluye el radiofónico. A los mayores les interesan, por tanto, las oportunidades que existen hoy día para contar cosas, y no solo ser parte de la audiencia, lo que requiere inevitablemente un proceso de aprendizaje.

La última dimensión del cuestionario es la que se refiere a los valores y la ideología, aspectos que, posiblemente, estén directamente relacionados con la baja confianza y el cuestionamiento de la veracidad informativa. No obstante, no solo se 
trata de valorar éticamente los medios, sino de ser conscientes de cómo los medios influyen en la percepción de la realidad que tiene la ciudadanía, implicándose en los procesos de construcción colectiva que permiten las herramientas 2.0. El impulso de la participación a través de Internet aparece como un verdadero estímulo para el envejecimiento activo. No se debe olvidar que, sea cual sea la vía de participación, el objetivo principal debe estar basado en mejorar el compromiso ciudadano.

En resumen, en lo que se refiere a la capacidad de análisis de este sector poblacional, la sabiduría que otorga su experiencia compensa en cierta forma la carenciade conocimientos técnicos o lingüísticos. Sin embargo, cada una de las dimensiones de la competencia mediática adolece de una mejora en las posibilidades de expresión, directamente relacionadas con el uso y la inmersión en Internet y en las Redes Sociales, siendo necesario que se supere la idea exclusivista de la Red como fuente de información. Cerca del 70\% no utiliza Internet para desarrollar una ciudadanía activa. Es conveniente, por tanto, dar un paso más allá del uso de los medios limitado a la información y a la creación de opinión, y apostar por la dimensión creativa.

El acceso a tecnologías y redes es, por tanto, mejorable en la población mayor de Andalucía, lo que influye directamente en el grado de competencia mediática. El sector analizado valora las posibilidades de comunicación de las que se dispone hoy día, aunque tiene desconfianza ante la veracidad de los medios institucionalizados. En las personas mayores, el interés y la actitud crítica constituyen una base idónea para fomentar la competencia mediática e impulsar la consolidación de una ciudadanía más activa, más aún si se tiene en cuenta el protagonismo de las personas con más de 55 años en la sociedad futura.

\section{Bibliografía}

COWGILL, Donald. (1974). Aging and modernization: A revision of the theory, en GUBRIUM, Jaber F. (ed.) Late life: Communities and environmental policy. Págs. 123-146. Charles C. Thomas. Springfield (EE.UU).

CORBETTA, Piergiorgio (2003): Metodología y técnicas de investigación social. Mcgraw Hill. Madrid.

CUMMING, Elaine \& HENRY, William (1961): Growing Old: The process of disengagement. Basic Books Inc. Nueva York.

FERRÉS, Joan (2007): La competencia mediática en comunicación audiovisual, en Comunicar, $n^{\circ}$ 29. Págs. 100-107.

FUNDACIÓN TELEFÓNICA (2013): La sociedad de la información en España 2012. Disponible en http://e-libros.fundacion.telefonica.com/sie12/. Consultado el 28 de junio de 2013.

GABELAS, José Antonio; MARTA-LAZO, Carmen \& ARANDA, Daniel (2012): Por qué las TRIC y no las TIC, en COMeIN. Revista de los Estudios de Ciencias de la Información y de la Comunicación, $\mathrm{n}^{\circ}$ 9. Disponible en 
GARCÍA MÍNGUEZ, Jesús (2004): La educación en personas mayores. Ensayo de nuevos caminos. Narcea Ediciones. Madrid.

HIDALGO, Jorge (2001): El envejecimiento. Aspectos sociales. Universidad de Costa Rica. San José (Costa Rica).

HERNÁNDEZ PINA, Fuensanta (1997): Conceptualización del proceso de la investigación educativa, en BUENDÍA, Leonor; COLÁS, Pilar \& HERNÁNDEZ PINA, Fuensanta: Métodos de investigación en Psicopedagogía. McGraw-Hill. Madrid.

LÓPEZ DOBLAS, Juan \& DÍAZ CONDE, Pilar (2013): La modernización social de la vejez en España, en Revista Internacional de Sociología, vol 71, $\mathrm{n}^{\circ} 1$. Págs. 65-89. Doi: 10.3989/ris.2011.04.26.

PÉREZ SERRANO, Gloria (coord.) (2004): Calidad de vida en personas mayores. Dykinson. Madrid.

PRENSKY, Marc (2001): Digital Natives, Digital Inmigrants. On the Horizon, en MCB University Press, vol 9, $\mathrm{n}^{\circ}$ 5. Octubre 20012. Disponible en http://www. nnstoy.org/download/technology/Digital\%20Natives\%20-\%20Digital\%20Immigrants.pdf. Consultado el 12 de julio de 2013.

SANTIBÃNEZ VELILLA, Josefina; RENÉS ARELLANO, Paula \& RAMÍREZ GARCÍA, Antonia (2012): Ciudadanía y competencia audiovisual en La Rioja: Panorama actual en la tercera edad, en Revista ICONO14, vol 10, $\mathrm{n}^{\circ} 3$. Disponible http://www.icono14.net/ojs/index.php/icono14/article/view/195. Consultado el 28 de junio de 2013.

SCOLARI, Carlos (2008): Hipermediaciones. Elementos para una teoría de la comunicación digital interactiva. Gedisa. Barcelona.

7.1 Artículos en publicaciones web:

CONSEJERÍA DE ECONOMÍA, INNOVACIO'N, CIENCIA Y EMPLEO DE LA JUNTA DE ANDALUCÍA. INSTITUTO DE ESTADÍSTICA Y CARTOGRAFÍA DE ANDALUCÍA (2013): Anuario Estadístico de Andalucía 2013. Apartado 2.2.2: "Población proyectada según grupos quincenales de edad y sexo por provincia. Años 2013 a 2035". Disponible en http://www.juntadeandalucia.es/ institutodeestadisticaycartografia/anuario/anuario13/cap02/anuario13-2_2_02. xls. Consultado el 25 de junio de 2013.

http://www.uoc.edu/divulgacio/comein/es/numero09/articles/Article-Dani-Aranda. $\mathrm{html}$. Consultado el 25 de junio de 2013.

ORGANIZACIÓN MUNDIAL DE LA SALUD (2012): Estadísticas sanitarias mundiales. Ginebra (Suiza). Disponible en http://apps.who.int/iris/bitstream/10665/44858/1/9789243564449_spa.pdf. Consultado el 26 de junio de 2013.

\section{Notas}

Esta investigación forma parte de la convocatoria para Proyectos de Excelencia de la Junta Andalucía, con clave P10SEJ-5823, titulado «La competencia audiovisual de la ciudadanía andaluza. Estrategias de alfabetización mediática en la sociedad del ocio digital ALFAMED». 


\section{Los autores}

Paloma Contreras-Pulido. Periodista y Educadora social. Máster en comunicación y educación audiovisual. Miembro del Grupo de Investigación Ágora de la Universidad de Huelva y del Grupo Comunicar. Docente de la asignatura "La comunicación como servicio público: La democratización de los medios" del Máster de Comunicación y Educación Audiovisual (UHU-UNIA).

Rafael Marfil-Carmona. Periodista e investigador en Comunicación Audiovisual y Publicidad en la Universidad de Granada. Profesor de la Escuela Superior de Comunicación y Marketing de Granada. Máster en Artes Visuales y Educación. Miembro del Grupo Comunicar y del Grupo de Investigación CiberAV "Cibercultura, procesos comunicativos y medios audiovisuales" (SEJ-058) de la Universidad de Granada.

Juana $\mathrm{M}^{\mathrm{a}}$ Ortega. Profesora Titular del Departamento de Pedagogía de la Universidad de Jaén. Docente de la asignatura Nuevas tecnologías aplicadas a la educación. Miembro del Grupo Comunicar y del Grupo de Investigación DIEA "Diseño e Investigación en Educación en Andalucía" (HUM-427) de la Universidad de Jaén. 